Note

\section{Saponin Composition in Developing Soybean Seed (Glycine max (L.) Merrill, cv. Mikuriyaao)}

\section{Makoto Shimoyamada, Kyuya Harada* and Kazuyoshi OKuBo}

Department of Food Chemistry, Faculty of Agriculture, Tohoku University, 1-I Amamiyamachi Tsutsumidori, Aoba-ku, Sendai 981, Japan

* National Institute of Agrobiological Resources, 2-1-2 Kannondai, Tsukuba 305, Japan

Received October 16, 1990

Saponins, which have many physiological activities, have been isolated from soybean seeds. ${ }^{1-8)}$ In previous papers, ${ }^{9,10)}$ we reported the saponin composition of soybean plants and germinating seeds. We have now analyzed the variation of saponins in developing soybean seeds.

Shiraiwa et al. reported that most varieties of soybean have $\mathrm{Aa}$ (acetyl-soyasaponin $\mathrm{A}_{4}$ ) and/or $\mathrm{Ab}$ (acetylsoyasaponin $A_{1}$ ) in the seed hypocotyl ${ }^{11}$ and that these constituents were controlled by codominant allelic alternatives at a single locus. ${ }^{12)}$ However, only Mikuriyaao lacks both $\mathrm{Aa}$ and $\mathrm{Ab}$, and contains $\mathrm{Af}$ (acetyl-soyasaponin $\mathrm{A}_{2}$ ) and $\mathrm{Ac}$ instead (Fig. 1). ${ }^{11)}$ It is noteworthy that only Mikuriyaao have an unusual saponin composition. Study- ing the saponins in an unisual mutant like this, some important informations would be obtained on the biosyntheses of saponins, the physiological significances of saponins, and the contribution of saponins to undesirable taste of soybean processed foods. These informations are useful to improve the acceptability of soybeans by breeding. So the saponin composition in developing soybean seeds (Glycine $\max$ (L.) MERRILL, cv. Mikuriyaao) were analyzed by HPLC (UV 205 nm) as reported in previous papers. ${ }^{9,10)}$

The fresh and dry weights of developing seed are shown in Fig. 2. The fresh weight rapidly increased until seed thickness reached $7.5 \mathrm{~mm}$ (about 55 days after flowering; $0.49 \mathrm{~g}$ ), then decreased because of dehydration. The dry weight also increased similarly, but subsequently remained constant until maturation.

Saponin constituents were separately analyzed in the hypocotyl and cotyledon. In the hypocotyl, soyasaponin I, Ac, and Af were detected. Each saponin level (\% dry weight) increased initially, reached a maximum and gradually decreased until seed maturation (Fig. 3). However, the changing points of each constituent were somewhat different. Namely, soyasaponin I level reached a maximum fastest at $3.5 \mathrm{~mm}$ thickness (about 25 days after flowering), then Ac level at $5.5 \mathrm{~mm}$ (about 40 days), at last Af level reached it at $6.5 \mathrm{~mm}$ (about 50 days). Further the Af level was much higher than any other constituents ( $\max .2 .3 \%$ ). Each saponin content (per hypocotyl) increased significantly, then remained constant till maturation. It is not thus considered that saponin contents in hypocotyl decrease during maturation.

In the cotyledon (Fig. 4), soyasaponin II as well as soyasaponin I, Ac, and Af were detected. The levels of soyasaponin I, Ac, and Af were were much lower than
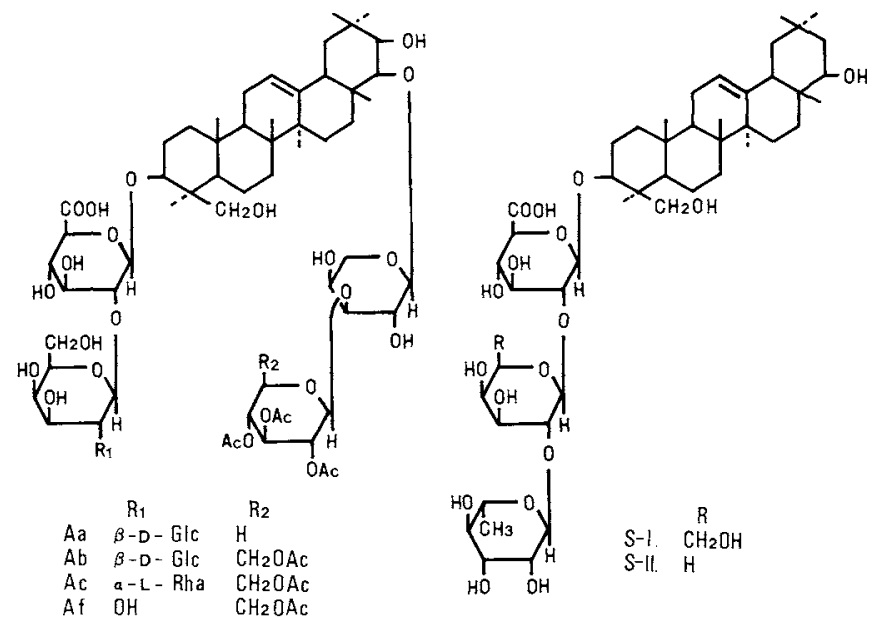

Fig. 1. Structures of Soybean Saponins.

Abbreviations: $\beta$-D-Glc, $\beta$-D-Glucopyranosyl; $\alpha$-L-Rha, $\alpha$-L-Rhamnopyranosyl; S-I, soyasaponin I; S-II, soyasaponin II. Mikuriyaao seeds have Ac, Af, and soyasaponins I and II. 
those in the hypocotyl; soyasaponin I was $1 / 6$ of that in the hypocotyl (at $3.5 \mathrm{~mm}$ ), Af was $1 / 50$ (at $6.5 \mathrm{~mm}$ ), and Ac was $1 / 70$ (at $7.5 \mathrm{~mm}$ ). Each saponin level in the

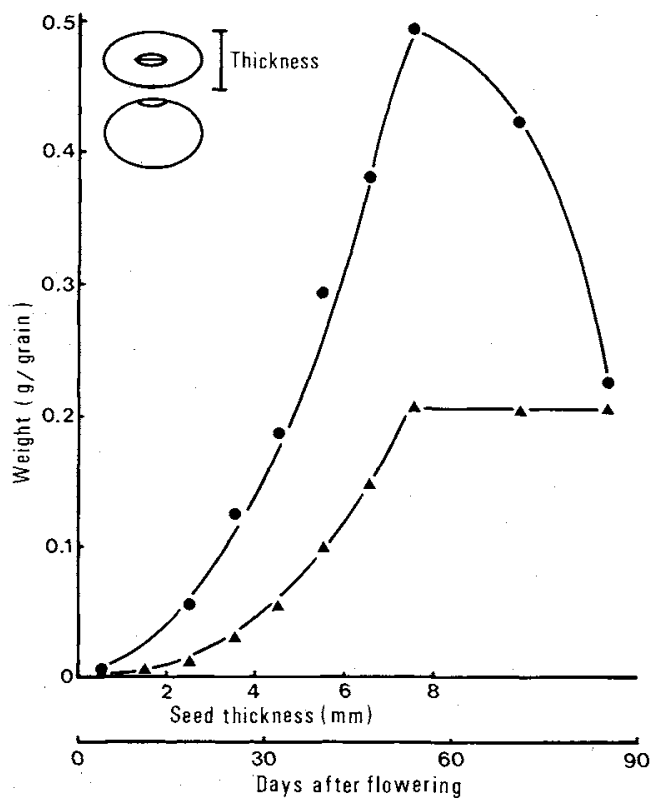

Fig. 2. Changes in Fresh and Dry Weights of Developing Soybean Seed.

$\boldsymbol{O}$, wet weight; $\boldsymbol{\Delta}$, dry weight.

The abscissa represents the thickness of immature seed, and the days after flowering was co-plotted. The inset shows the seed thickness of soybean seed.

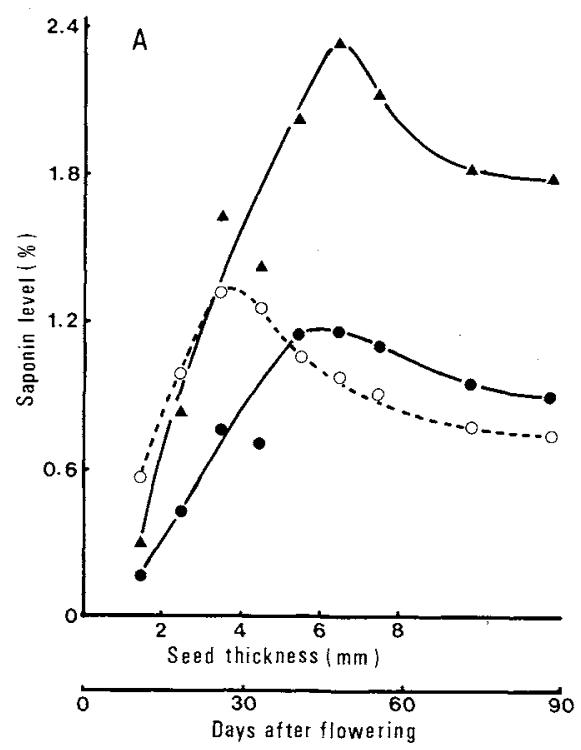

cotyledon varied in a similar manner to those in the hypocotyl during maturation. Soyasaponins I and II and Ac levels decreased significantly, but the Af level remained almost constant through to the mature seed. The changing points of Ac and Af were a little faster than those in the hypocotyl ( $3.5 \mathrm{~mm}$; about 25 days after flowering). The increase of soyasaponin. II level were continued till later than the other saponins in the cotyledon $(4.5 \mathrm{~mm}$; about 30 days).

Regarding the saponin content (per pair of cotyledons), each saponin initially increased. At first the soyasaponin II content reached a maximum at $5.5 \mathrm{~mm}$ (about 40 days; $62 \mu \mathrm{g}$ ), then the contents of soyasaponin I, Ac, and Af reached maxima at $6.5 \mathrm{~mm}$ (about 50 days; 85,25 , and $55 \mu \mathrm{g}$, respectively). Then the increases of the saponin contents stopped. This changing point is almost equal to that in hypocotyl. In the developing soybean seed, it was shown that the saponin accumulation is completed before the endpoint of fresh and dry weight increases.

Shiraiwa et al. ${ }^{11)}$ studied the saponin composition in many varieties of soybean seed hypocotyl, and reported that only Mikuriyaao had Ac and Af instead of the usual acetylated saponins; through most varieties had $\mathrm{Aa}$ and/or $A b$. It is considered that Af is a desglucosyl Ab, and occurs mainly in the hypocotyl, being detected in small amounts in other $\mathrm{Ab}$ type varieties. ${ }^{13)}$ Mikuriyaao is hence likely to be a variety lacking a glucosyltransferase that catalyzes the glucasylation of a galactosyl moiety of Af.

\section{References}

1) I. Kitagawa, H. K. Wang, T. Taniyama and $M$. Yoshikawa, Chem. Pharm. Bull., 36, 153 (1988).

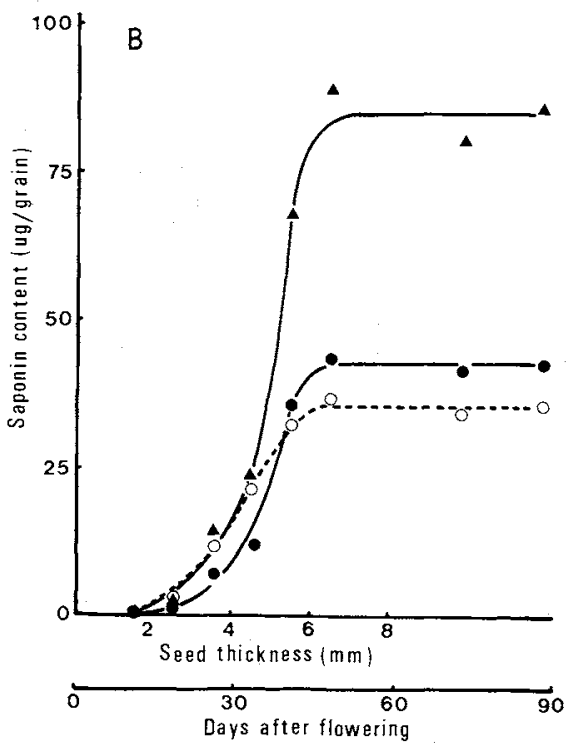

Fig. 3. Variation of Saponins in Developing Soybean Seed Hypocotyl.

A, saponin level (\% dry weight); B, saponin content ( $\mu$ g/grain). $\boldsymbol{O}, \mathrm{Ac} ; \boldsymbol{A}, \mathrm{Af} ; \mathrm{O}$, soyasaponin I. 

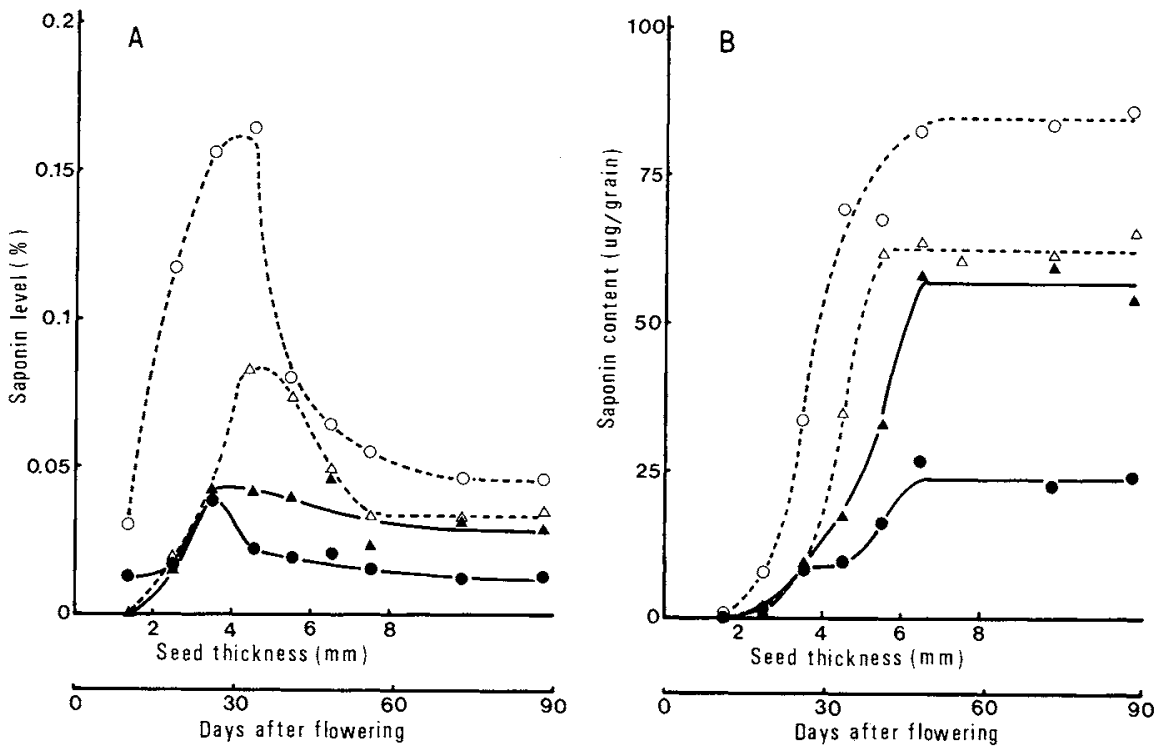

Fig. 4. Variation of Saponins in Developing Soybean Seed Cotyledon.

A, saponin level ( $\%$ dry weight); B, saponin content ( $\mu$ g/grain). soyasaponin II.

2) J. C. Burrows, K. R. Price and G. R. Fenwick, Phytochemistry, 26, 1214 (1987).

3) C. L. Curl, K. R. Price and G. R. Fenwick, J. Sci. Food Agric., 43, 101 (1988); T. Taniyama, M. Yoshikawa and I. Kitagawa, Yakugaku Zasshi, 108, 562 (1988).

4) I. Kitagawa, M. Saito, T. Taniyama and M. Yoshikawa, Chem. Pharm. Bull., 33, 1069 (1985).

5) I. Kitagawa, M. Saito, T. Taniyama and $M$. Yoshikawa, Chem. Pharm. Bull., 33, 598 (1985).

6) C. L. Curl, K. R. Price and G. R. Fenwick, J. Natural Prod., 51, 122 (1988).

7) I. Kitagawa, T. Taniyama, Y. Nagahama, K. Okubo, F. Yamauchi and M. Yoshikawa, Chem. Pharm. Bull., 36, 2819 (1988).
8) T. Taniyama, Y. Nagahama, M. Yoshikawa and I. Kitagawa, Chem. Pharm. Bull., 36, 2829 (1988).

9) M. Shimoyamada, S. Kudo, K. Okubo, F. Yamauchi and K. Harada, Agric. Biol. Chem., 54, 77 (1990).

10) M. Shimoyamada and K. Okubo, Agric. Biol. Chem.. 55, 577 (1991).

11) M. Shiraiwa, M. Shimoyamada, K. Okubo, F Yamauchi, M. Yoshikawa and I. Kitagawa, Proc. Int Symp. on New Technology of Vegetable Proteins, Oils and Starch Processing, Beijing, China, Vol. 1, 1987 , p. 96.

12) M. Shiraiwa, F. Yamauchi, K. Harada and K. Okubo, Agric. Biol. Chem., 54, 1347 (1990)

13) unpublished data. 